Recibido: julio de 2014

Aprobado: agosto de 2014

\section{EL ANTIGUO HOSPITAL SAN JUAN DE DIOS. UNA VALORACIÓN HISTÓRICA PARA SU RECONOCIMIENTO COMO BIEN DE INTERÉS CULTURAL DE BUCARAMANGA*}

Román Javier Perdomo González**

DOI: http://dx.doi.org/10.15332/rev.m.v11i2.1722

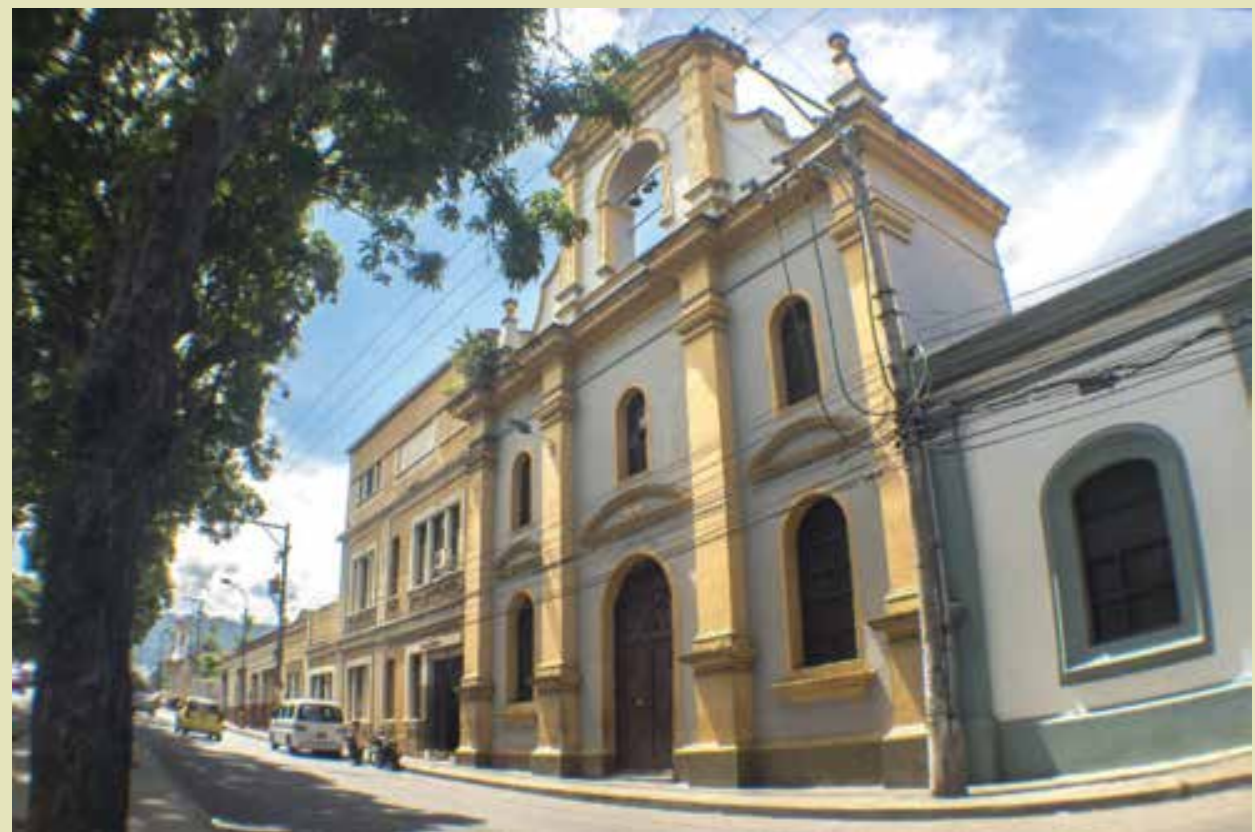

Bucaramanga

Fuente: Román Perdomo, 2014.

El presente artículo es derivado del trabajo de grado para obtener el título de historiador en la Universidad Industria de Santander UIS, dicha investigación se realizó con Sergio Acosta Lozano y So Sierra Flórez, dentro de las actividades del proyecto denominado Plan de Rescate y Aprovechamiento del Patrimonio Arquitectónico de Bucaramanga (PRAPAB) liderado por el área de patrimonio inmueble del Instituto Municipa de Cultura y Turismo de Bucaramanga, Colombia.

** Historiador de la Universidad Industria de Santander. Asociación Historia Abierta, Bucaramanga. Correo electrónico: rperdomog@gmail.com
En el presente artículo se hace una valoración histórica del edificio donde funcionó el antiguo Hospital San Juan de Dios de Bucaramanga. Se pretende con esto, aportar al reconocimiento de este inmueble como parte del patrimonio arquitectónico de la ciudad y su posterior inclusión en la lista de Bienes de Interés Cultural del municipio. Se presentan los hallazgos logrados mediante la investigación histórica, resaltando la importancia de esta edificación para la historia de Bucaramanga, teniendo en cuenta que fue la primera, y por largo tiempo, la única institución pública de salud que prestó sus servicios a los bumangueses.

\section{PALABRAS CLAVE}

Hospital San Juan de Dios, valoración histórica, patrimonio arquitectónico, bien de interés cultural, Bucaramanga. 


\section{THE ANCIENT SAN JUAN DE DIOS HOSPITAL. A HISTORICAL ASSESSMENT FOR ITS RECOGNITION AS HERITAGE OF CULTURAL INTEREST OF BUCARAMANGA}

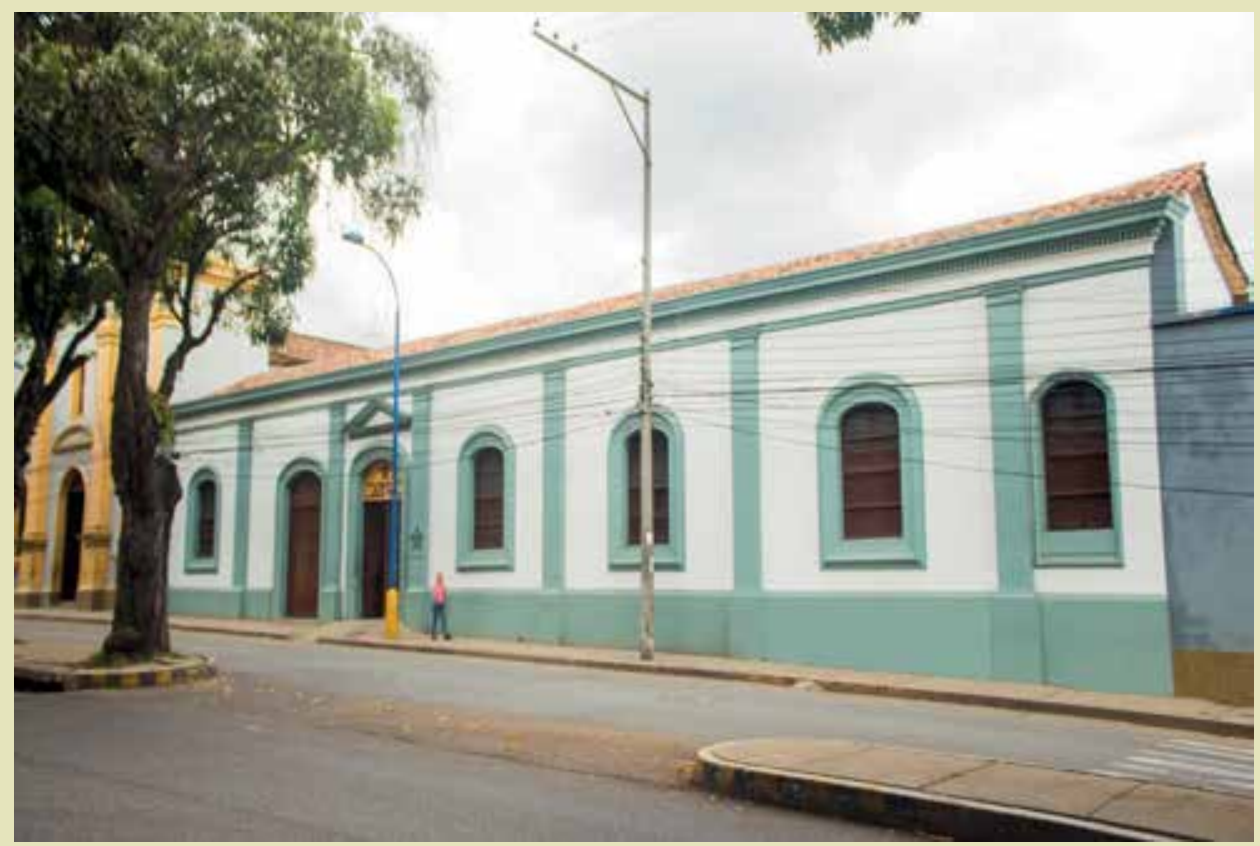

Fachada del edificio del antiguo Hospital San Juan de Dios

Fuente: Román Perdomo, 2014

\section{ABSTRACT}

This article is a historical valuation of the building of the old Hospital San Juan de Dios de Bucaramanga. It seeks to contribute to the recognition of the building as an architectural heritage of the city, and its inclusion in the list of Cultural Interest. The findings made by historical research are presented, highlighting the importance of this building for history of Bucaramanga, considering it was the first, and for a long time, the only public health institution that served the residents of Bucaramanga.

\section{KEYWORDS}

Hospital San Juan de Dios, historical valuation, architectural heritage, cultural interest, Bucaramanga. 


\section{INTRODUCCIÓN}

La Ley I I 85 de 2008 y el Decreto reglamentario número 763 de 2009 definen con claridad los criterios de valoración marco que contribuyen a la atribución y definición de la significación cultural, tanto de los bienes muebles como de los inmuebles. Estos valores marco son tres: el valor histórico, el valor estético y el valor simbólico. Estos valores se convierten en herramientas interpretativas que permiten, entre otras cosas, establecer si un bien es susceptible de ser considerado como un Bien de Interés Cultural:

Cuando se alude al patrimonio cultural, estos tres valores marco, en su concepción amplia, siempre están presentes y se entrelazan entre sí. Pueden reconocerse, hacerse visibles y explícitos en la formulación y aplicación de criterios que concreten su significado en la particularidad de los bienes culturales. En la valoración de unos objetos se hará énfasis en el valor simbólico y, en otros, se hará en el valor estético o en el histórico, dependiendo de los tiempos y las condiciones socioculturales (López, García \& Medina, 2005, p. 38).

El valor estético es un atributo que puede referirse a la calidad artística y de diseño de un bien, por lo cual, se relaciona siempre con las características físicas y formales que lo conforman. Dicho atributo puede reflejar "(...) una idea creativa en su composición, en la técnica de elaboración o construcción" (Ministerio de Cultura, 2009, p. 38), de ahí que resulte importante preservar. Por su parte, el valor simbólico se refiere a la vinculación de un determinado bien con "procesos, prácticas, eventos o actividades significativas para la memoria o el desarrollo constante de la comunidad" (Ministerio de Cultura, 2009, p. 38). Lo simbólico no se debe entender como algo impuesto, único o establecido, sino como un atributo en constante transformación.

En cuanto al criterio de valoración de orden histórico, ha tenido una evolución conceptual a través del tiempo y en la actualidad se entiende en un sentido amplio. No se trata de valorar un bien solo por su antigüedad o por su resistencia al paso del tiempo, sino más bien por el significado e importancia que tiene en la historia de una sociedad, ya sea de orden nacional, regional o local: "es la asociación directa del bien con épocas, procesos, eventos y prácticas políticas, económicas, sociales y culturales” (Ministerio de Cultura, 2009, p. 38). En este sentido, un bien patrimonial tiene la característica de convertirse en un documento para la historia, en testigo de acontecimientos del pasado, en parte de nuestra identidad y en un elemento que activa la memoria de la comunidad.

De este modo, la historia es el insumo permanente para la construcción y el mantenimiento de la memoria. Es necesaria para identificar y poner en valor nuevos bienes susceptibles de ser incluidos dentro de la lista de Bienes de Interés Cultural, como es el caso del antiguo Hospital San Juan de Dios de Bucaramanga. En el caso del patrimonio arquitectónico, conocer la historia de una edificación y de la institución que lo acoge, debería ser uno de los primeros pasos para seguir si se quiere lograr la identificación y apropiación de nuevas manifestaciones del patrimonio cultural. Lo anterior responde a una premisa general, la cual indica que ninguna comunidad valora lo que no conoce, de ahí la importancia de la investigación histórica como herramienta para la identificación del patrimonio cultural.

En las siguientes páginas se quiere dar a conocer una breve reseña histórica del antiguo Hospital San Juan de Dios, con el objetivo de aportar al reconocimiento de este bien inmueble como parte de nuestro patrimonio arquitectónico, pues en la actualidad no se 
encuentra declarado como tal. No está de más remarcar que el enfoque se centra en el reconocimiento del valor histórico del bien, quedando el análisis de su valor simbólico y su valor estético para futuras investigaciones.

\section{RESEÑA HISTÓRICA DEL HOSPITAL SAN JUAN DE DIOS}

El antiguo Hospital San Juan de Dios de Bucaramanga tiene su origen en la primera mitad del siglo XIX. De acuerdo con Roberto Harker Valdivieso (1977), la construcción del primer edificio que sirvió de hospital en esta ciudad se inició el $I^{\circ}$ de mayo de 1845. Carlos Humberto Espinosa (1996) coincide en afirmar que la construcción del primer edificio para hospital se inició con anterioridad a 1850 , sin embargo, fue puesto al servicio del público solo hasta el 8 de marzo de 1853. Lo anterior sugiere la existencia de un periodo prolongado de construcción, interrumpido por inconvenientes de tipo económico que, como veremos, pudieron finalmente resolverse gracias a la participación activa de la comunidad.

La iniciativa de construir una edificación que sirviera como hospital nació por parte de la misma sociedad bumanguesa. Sin embargo, fue gracias al esfuerzo y liderazgo del sacerdote José lgnacio Martínez lo que permitió la realización del proyecto, estimulando y organizando actividades en procura de la consecución de recursos económicos para la obra. Así, "el buen pastor de almas reunía en su casa a los notables del poblado para decirles de la necesidad de un hospital, organizaba veladas líricas, bazares, en fin, cuántos recursos fueron dables para obtener fondos a su obra" (Acevedo, 1953, p. 18).

El cronista José Joaquín García (1992) dejó testimonio de la forma cómo se financió la construcción del hospital: "Para arbitrar recursos resolvieron organizar algunas representaciones teatrales, en la misma casa del cura, y para ello tomaron parte activa en la empresa algunos jóvenes y varias personas de la familia Martínez Nieto" (p. 134). Pero los dineros recolectados producto de estas actividades no fueron suficientes para terminar la obra iniciada en 1845 , así "se dio a los trabajos, pero luego quedaron suspendidos por no poco tiempo, hasta que en 1853 se organizaron de nuevo, apelando a los mismos medios que antes para seguir adelante la empresa" (García, 1992, p. 134).

Años más tarde, el apoyo dado por el sacerdote José lgnacio Martínez a las obras del hospital fue reconocido en la Gaceta del Santander en noviembre 1870:

También es digna de todo elojio la conducta del venerable Cura párroco i de los médicos de esta ciudad; la del primero, por el impulso que ha dado al hospital de caridad, con su influencia, con sus propios recursos i con su jenial decisión por las obras públicas; i la de éstos, por los importantes servicios que prestan a los enfermos en el ejercicio de su misión; así como también lo es la del boticario, señor Guillermo Müller, quien suministra gratuitamente los medicamentos para el hospital de Bucaramanga (pp. 509-5 I0).

El Hospital San Juan de Dios se construyó en un terreno ubicado en la cuarta manzana al sur de la entonces "Plaza Principal", justo al lado del Cementerio Central, enfrente del Parque Romero'. Dichos terrenos fueron donados por Facundo Mutis y Juan Crisóstomo

I Aunque en ese tiempo este parque no existía, por mucho tiempo al espacio urbano que había en frente del hospital se le conoció como la Plazuela del Hospital “[...] apenas un potrero descubierto" el mismo espacio que años más tarde se convertiría en el Parque Romero, tras la donación de los terrenos por parte de Trinidad Parra de Orozco y de Anselmo Peralta (Espinosa, 1996, p. 34). 
Parra, dos miembros reconocidos de la elite local y propietarios de varios predios urbanos (Espinosa, 1996).

Figura I. El Parque Romero (entorno urbano del Hospital San Juan de Dios de Bucaramanga) Fuente: Autor desconocido. Parque Romero de Bucaramanga. [Fotografía], Bucaramanga [Online] Grupo de Facebook: Fotos de Bucaramanga (antiguas, modernas y actuales). Consultado el 10 de diciembre de 2013

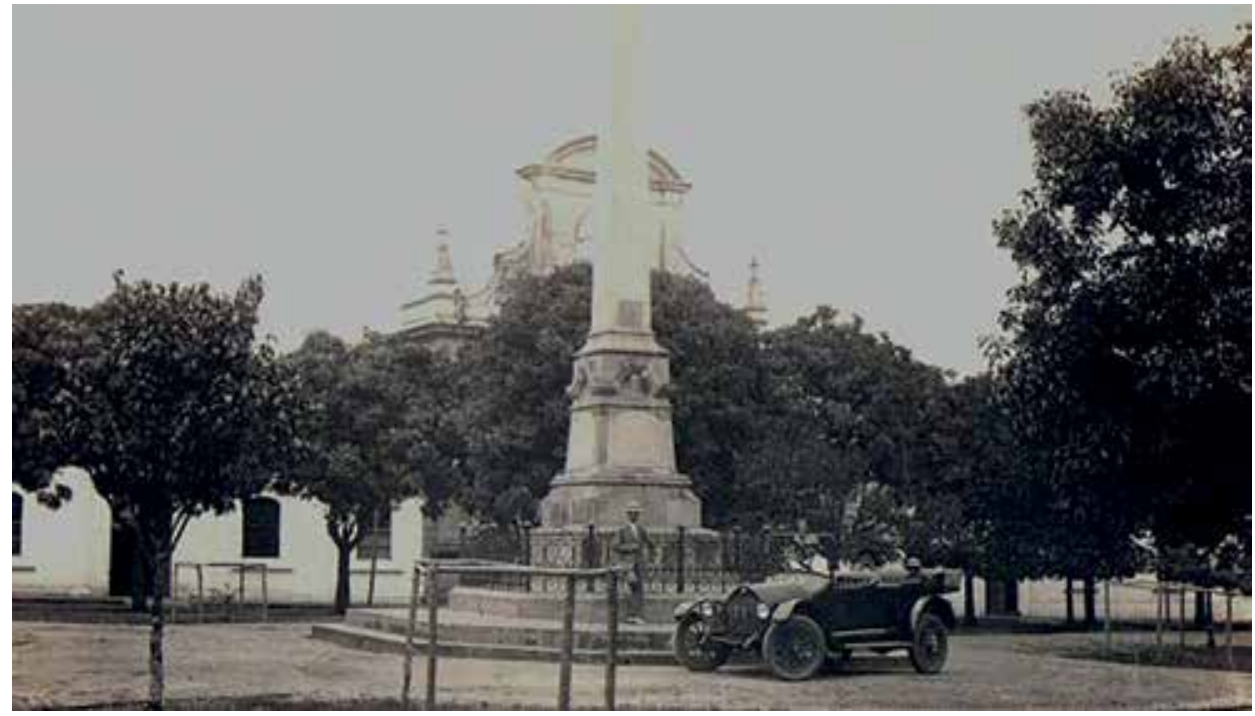

En sus primeros años de funcionamiento la edificación era muy pequeña y modesta. Estaba construida en tapia pisada, piso de tierra y techo de teja, Spinel (2009) afirma "...no tenía un espacio suficiente sino para albergar a unos pocos enfermos” (p. 65). Esta edificación tenía más o menos las siguientes características el año de su apertura:

(...) tenía elementos arquitectónicos como la capilla que le daba un toque más religioso que de centro para la atención de los enfermos y estaba compuesta por dos salas, con corredores al norte y al sur, una para los hombres y otra para las mujeres. Dos piezas más para el servicio de la enfermera y para la preparación de los alimentos (Espinosa, 1996, p. 187).

En cuanto a la dotación necesaria para el funcionamiento del hospital, se conoce que no se contaba con los elementos básicos para tal fin como personal capacitado, medicamentos y áreas con buenas condiciones de higiene para atender a los bumangueses y poblaciones vecinas. Lo anterior debido en gran medida a que tanto la infraestructura física y los trabajadores del hospital no estaban preparados para afrontar una emergencia de gran magnitud, como las epidemias de viruela que se presentaron en la ciudad. Por su parte, la mayoría del personal no contaba con estudios especializados, la profesión la habían aprendido de manera empírica por medio de la observación y la práctica constante. En el caso de los medicamentos, estos solo podían adquirirse en las boticas ubicadas en la Calle del Comercio (hoy calle 34) y no pasaban de ser paliativos para dolencias comunes como el dolor de muela o de cabeza (Spinel, 2009).

Entre los primeros funcionarios del Hospital figuraban tres enfermeras, huéspedes del hospital, a quienes recuerda José Joaquín García (1992) de una manera muy particular: "[...] sus nombres eran Josefina Trigueros, una 'tullida' llamada Rita y una 'manca' llamada Liberata" (p. 134 - 135). El primer médico -y el único por muchos años- fue el doctor Aparicio Reyes "infatigable servidor de los humildes y de los enfermos", quien estuvo en el cargo por más de 20 años desde la apertura del hospital en 1853. Los oficios espirituales para los enfermos estuvieron a cargo del sacerdote Rafael Almansa (Acevedo, 1953). 
Más adelante, hacia 1865 , el Hospital sufrió una reforma administrativa que permitió el recibo de mendigos que habitan las calles de la ciudad, transformándolo en una institución de beneficencia, en la cual se atendía a los pacientes enfermos desamparados, que por una u otra razón no podían costearse los servicios médicos (García, 1992; Espinosa, 1996).

La necesidad de centros de salud y hospitales se hacía indispensable, más aún cuando en ese periodo enfermedades como la elefantíasis y la lepra parecían muy comunes en la Provincia de Soto. En efecto, tal y como se puede observar en una nota aparecida en la Gaceta de Santander de 1870:

La elefantiasis se propaga en las poblaciones de Soto de un modo aterrador, i se hace cada día más imperiosa la necesidad de crear un nuevo Lazareto para recoger a los desgraciados leprosos que vagan por las calles i caminos públicos, i son una amenaza para la parte sana de la población (pp. 509-5I0).

Además de prestar un servicio asistencial a los enfermos, en el hospital no se dejaba de lado la parte espiritual, justificándose la presencia de la Capilla para los oficios religiosos y donde la remodelación y decoración se contraponían a las dos piezas o locales para atender a los enfermos que contaban con una estructura física y de salubridad poco adecuados y los enfermos eran atendidos con un mínimo de cuidados médicos, porque, como ya se anotó, los profesionales en este campo eran escasos (Espinosa, 1996).

Hacia I87I el doctor Aparicio Reyes "respetable y antiguo medico" de Bucaramanga, promovió una serie de obras que buscaban la ampliación del hospital. Para ello solicitó la ayuda de amigos y donaciones voluntarias, por medio de las cuales y en el transcurso de dos años obtuvo los recursos financieros para construir un nuevo espacio para las enfermeras, así como más habitaciones y adecuar la capilla del hospital. Si bien no se tienen datos sobre las características arquitectónicas de la capilla y qué tipo de mejoras se incorporaron, sí se tiene conocimiento de su ubicación junto al hospital. Veinte años más tarde, esta misma capilla volvió a ser reconstruida, pero en dicha oportunidad fue el párroco José María Villalba el abanderado de la nueva obra (Spinel, 2009).

En 1893 se encargó al señor Joaquim Wilches, ingeniero de profesión, el dibujo del plano correspondiente a la nueva estructura, presupuestándose una suma de $\$ 80.000$ para su ejecución, y contando con el apoyo del Gobierno local y la Asamblea Departamental (García, 1992). Es así como el hospital y su nueva capilla, junto con el cementerio y el futuro surgimiento de un espacio público emblemático como lo fue el Parque Romero, justo frente a estas edificaciones, lograron hacer de este uno de los sectores más representativos dentro de la trama urbana de la ciudad de Bucaramanga a partir de la segunda mitad del siglo XIX.

Así, el "barrio del Cementerio y el Hospital" fue reconocido por mucho tiempo como uno de los sectores más bellos con los cuales contaba Bucaramanga, en el cual podría encontrarse "un escogido lugar de distracción y recreo donde pasar las fastidiosas horas de los días desocupados"2.

2 Recordemos que el Parque Romero, antes de llamarse así, se llamó Parque García Rovira. Posteriormente con la instalación de la estatua del prócer en la Plaza Principal, esta última tomó el nombre que actualmente lleva. "Parque García Rovira" (Semanario El Posta, 1894, p. 5). 
En 1888 el Hospital San Juan de Dios contó con la participación de las Hermanas de la Caridad, las cuales fueron traídas a la institución gracias a las gestiones de tres "damas de la ciudad": Trinidad Parra de Orozco, hija de Crisóstomo Parra, Zoila Uribe de Pradilla y Juana Ordoñez de Martínez. Así, a finales de 1888:

Hay alborozo en el poblado, las cuatro primeras religiosas de la caridad, las hermanas de la Presentación, Superiora, Manuela, Basilia y Enriqueta han llegado a hacerse cargo del Hospital al cual concurren personalmente aquellas señoras todos los días, i asisten a los enfermos con una constancia digna de ejemplo, cuidando de esos desgraciados como de sus propios hermanos (Gaceta de Santander, 1888, p. 493).

Los servicios prestados por las Hermanas de la Caridad se prolongaron por muchos años. Esta labor fue reconocida por la comunidad y el Gobierno local, ya que en 1953, la Junta de Beneficencia de Bucaramanga otorgó un reconocimiento a este grupo de religiosas por medio de una "Tarjeta de Oro", entregada a la madre Ana Esther, directora de las Hermanas de la Caridad durante las celebraciones del primer centenario del Hospital, como "reconocimiento a las labores que en los últimos 65 años ha venido prestando la benemérita institución a favor de los enfermos de nuestro Hospital”3.

Para finales del siglo XIX, la ciudad ya había tenido un crecimiento significativo. Gracias a su floreciente actividad comercial y artesanal de los años anteriores Bucaramanga se posicionó como el centro poblacional de mayor importancia tanto económica como política de la región, siendo designada capital del Departamento de Santander en I886, tras el final del periodo federal y la vuelta al centralismo. A finales de siglo XIX el Hospital San Juan de Dios se había posicionado como una institución destacada a nivel regional. Prueba de lo anterior, fue la designación en 1893 como sede de una reunión de médicos de la región que tuvo como objetivo constituir la Sociedad de Medicina de Santander (Acevedo, 1953).

Hasta 1890 el Hospital había funcionado mediante el sentido común, es decir, no había normas técnicas o personas nombradas o pagas que lo dirigiesen. Aunque no fue el objetivo de su establecimiento en Bucaramanga, las Hermanas de la Caridad tomaron desde un comienzo la dirección del hospital y organizaron su funcionamiento interno, centrando su atención primordialmente en los enfermos, ancianos, mendigos y desahuciados (Espinosa, 1996). Pero ya a finales del siglo XIX, el pensamiento de mejorar la salubridad del poblado comienza a aparecer entre la elite de la ciudad y a hacerse visible en las mejoras materiales:

[...] unido a la necesidad de construir puentes, empedrar las calles, refaccionar los edificios públicos y extender los caminos, hubo la necesidad de un mejoramiento en el estado sanitario y en las condiciones de salubridad de la población en general, que incluía el hospital, el asilo de locos, de huérfanos, ancianos o vagabundos, el cementerio, la plaza de mercado y el matadero (Spinel, 2009, p. 49).

Para la realización de estas obras se destinaron recursos provenientes del Gobierno departamental. Así, el 6 de julio 1894 en la Gaceta de Santander aparece publicada una Ordenanza de la Asamblea de Santander, en la cual se destinaban 300 pesos mensuales provenientes del Tesoro del Departamento "que se apropiarán en el Presupuesto de 1895 y 1896, para la reconstrucción y mejora del Hospital de Bucaramanga” (p. 5847). Las obras se iniciaron

3 "Hace cien años nació el Hospital San Juan de Dios de Bucaramanga" (Vanguardia Liberal, 1953). 


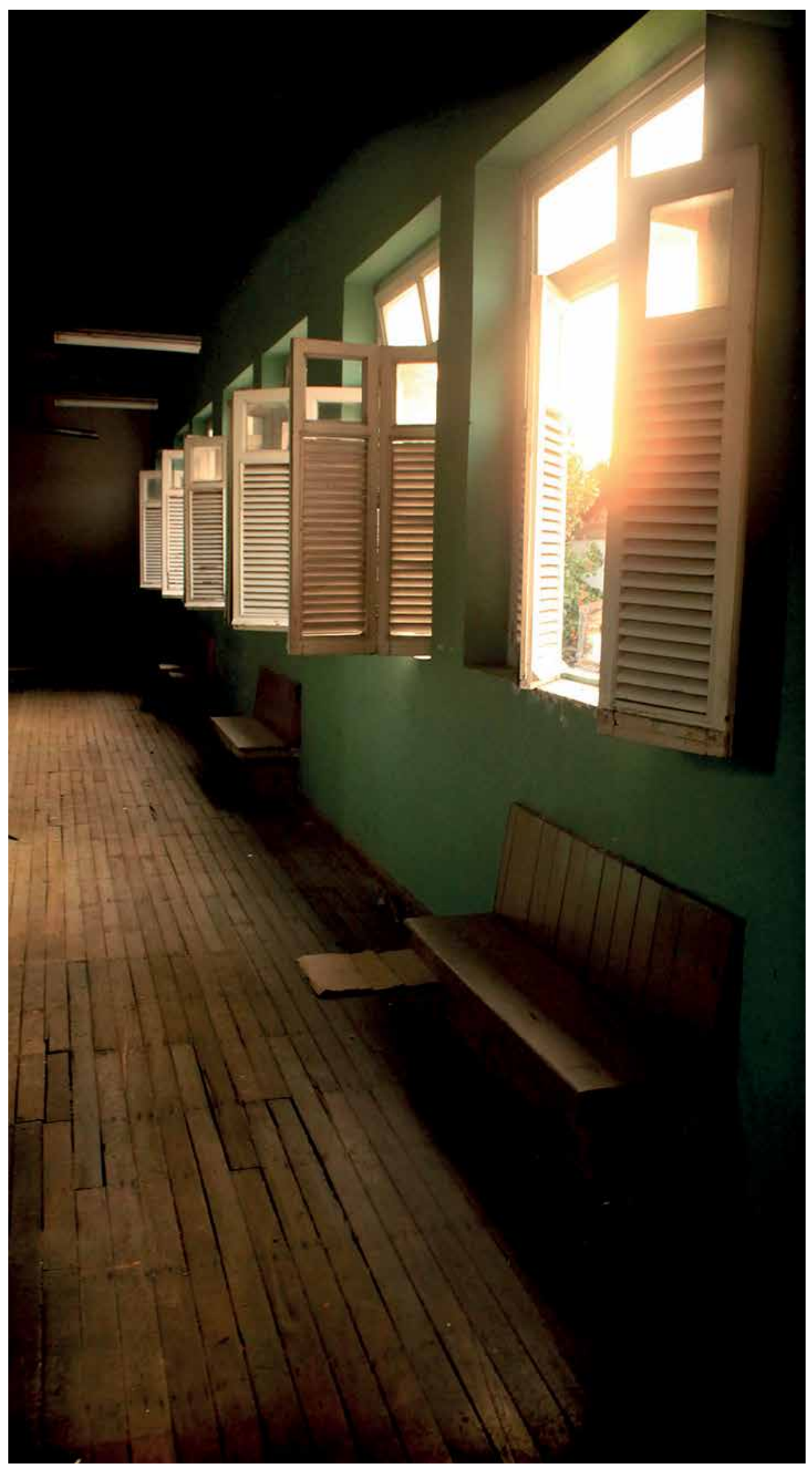

Figura 4. Interior del antiguo Hospital San

Juan de Dios de Bucaramanga

Fuente: Román Perdomo, 2014. 




REVISTA M VOL. 11 No.2. JULIO-DICIEMBRE 2014 • FACULTAD DE ARQUITECTURA • UNIVERSIDAD SANTO TOMÁS COLOMBIA M 32 
Figura 3. Interior del antiguo Hospital San

Juan de Dios de Bucaramanga

Fuente: Román Perdomo, 2014.

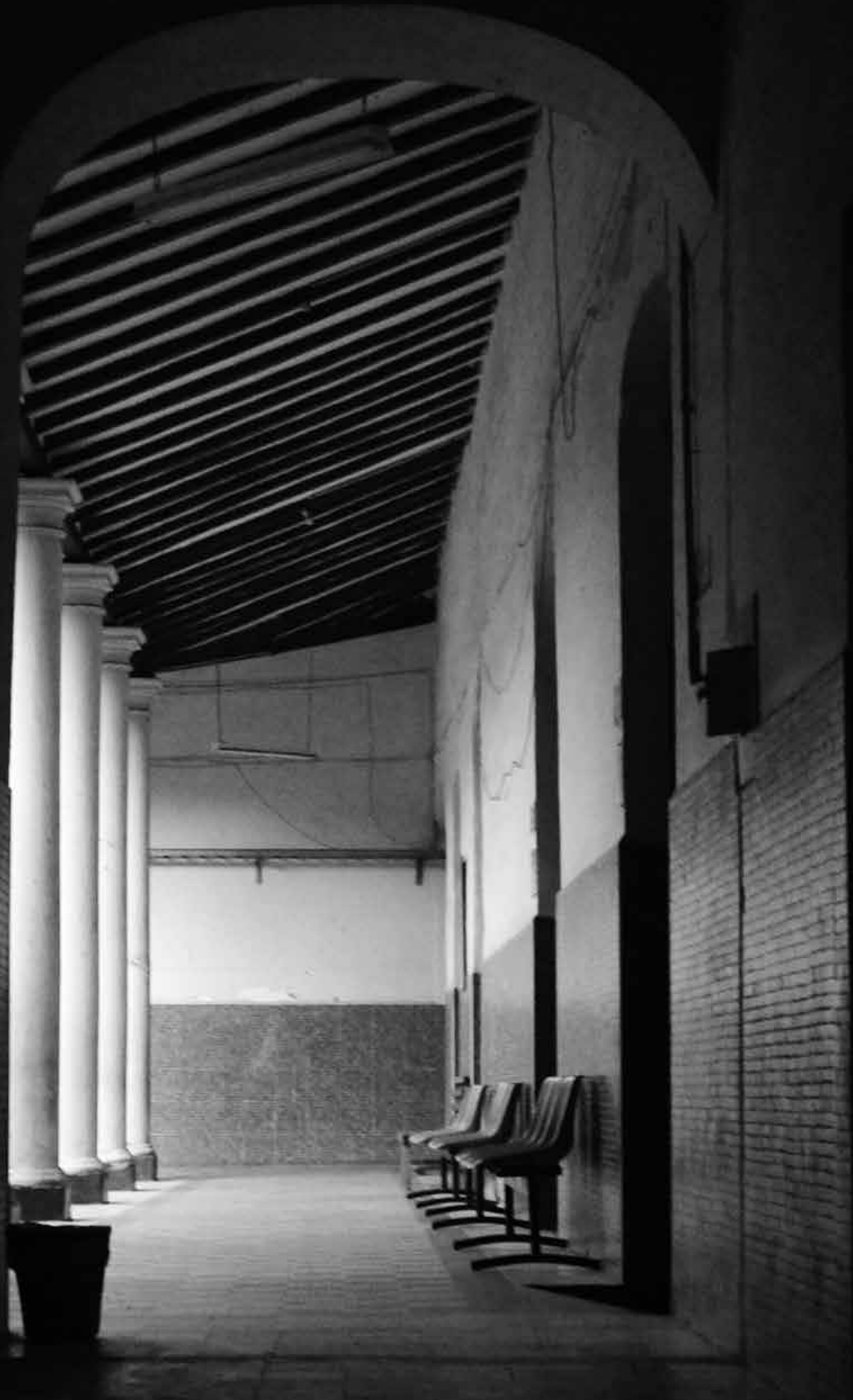


un año después, sin embargo, se vieron interrumpidas por los enfrentamientos bélicos de la Guerra de los Mil Días. Después de la finalización de la guerra, los trabajos se retomaron en I90I con el apoyo del gobernador de aquel entonces Ramón González Valencia y las generosas donaciones del general Prospero Pinzón, encargándose de la dirección de los trabajos al señor Paulino Colmenares, síndico del hospital (Revista Estudio, 1932, p. 160).

Resulta importante mencionar que el Hospital San Juan de Dios tuvo un papel importante en la atención de los hombres que resultaron heridos en la famosa batalla de Palonegro, ocurrida en una zona cercana a la ciudad, entre el 15 y 31 de mayo de 1900. Se calcula que el personal encargado atendió aproximadamente 1017 heridos, cifra que sobrepaso la capacidad del hospital y se hizo necesaria la adecuación de cinco casas como hospitales provisionales para sortear la emergencia. Para Mario Acevedo Díaz, director del Hospital San Juan de Dios en 1953, este fue el "momento más solemne" en la historia del Hospital (Acevedo, 1953).

A comienzos del siglo $X X$ y a medida que Bucaramanga se iba expandiendo físicamente y su población aumentaba, la deficiencia del Hospital en cuanto a los servicios que se prestaban y su infraestructura, se fueron haciendo cada vez más evidentes.

La incapacidad en cuanto a las instalaciones en las cuales funcionaba el Hospital derivaban en problemas de falta de espacios operativos y funcionales. La antigua edificación ubicada frente al Parque Romero "no daba abasto con tantos enfermos, diseminados en múltiples áreas y servicios” (Moreno, 2008, p. 66).

Tal cual ocurrió desde su apertura como institución prestadora de servicios de salud, el Hospital en la segunda década del siglo $X X$, aún requería para su sostenimiento en gran parte las ayudas prestadas por grupos de caridad y de "damas" importantes y de religiosos que alentaban a los bumangueses a "emprender campañas en pro de dicho Hospital, organizando para ello, tés bailables y bazares, debido a que las ayudas de las administraciones nunca fueron suficientes para evitar las condiciones extremas en las que allí se trabajaba" (Moreno, 2008, p. 67).

Teniendo en cuenta dicha situación, el Gobierno local empezó a preocuparse por la problemática concerniente a la salud pública, aunque los problemas de fondo no llegaron a solucionarse. Por ejemplo, la donación de 1000 pesos oro que hizo el Gobierno municipal en 1925, provenían de un traspaso de fondos que se había destinado en un principio a la fallida empresa del Teatro municipal. El dinero traspasado en aquella oportunidad se destinó finalmente a la compra de "[...] cien catres de hierro con destino al servicio del expresado establecimiento" (Vanguardia Liberal, 1925).

En los periódicos locales se anunciaba constantemente el problema y se advertía que la ciudad debía tener una edificación para un hospital moderno que pudiera responder las exigencias de los pobladores de la ciudad. Sin embargo,

[...] aunque la necesidad de construir un nuevo hospital fue una idea que cada vez tomaba más fuerza, debido a las constantes denuncias de la población a través de la prensa escrita, se optó por una solución transitoria, es decir, invertir recursos para realizar ampliaciones a la infraestructura decadente del ya existente con el objeto de dejarlo "mejor equipado" por algunos años más (Moreno, 2008, p. 69). 
En medio de este contexto, la idea de construir un nuevo hospital se hacía cada vez más fuerte. La propuesta de construir un edificio que respondiera arquitectónicamente a las necesidades de la institución se hizo oficial en 1946, para lo cual se dispusieron recursos en gran parte provenientes del Gobierno municipal, dentro de los cuales figuraba una contribución mensual de 50.000 pesos oro hasta el momento en que finalizaran los trabajos. (Moreno, 2008). Este fue el punto de partida de la llamada "nueva era hospitalaria" del San Juan de Dios. Aunque se trataba de la misma iniciativa una vez construida la nueva edificación para el hospital, este cambiaría su nombre. Ya para la década del setenta, la propuesta derivó en lo que se conoció años más tarde con el nombre de Hospital Ramón González Valencia, aunque esta nueva edificación tardaría muchos años más en concretarse:

[...] la creación del mencionado hospital fue un sueño que comenzó en 195 I pero se materializó hasta 1973 con el Acuerdo No. 10 del 30 de noviembre del mencionado año, en el cual se manifestaba que dado los estudios fiscales de la época era imposible sostener dos hospitales en la ciudad, el Hospital San Juan de Dios y el Hospital Universitario Ramón González Valencia y sumado a esto, el deterioro del antiguo hospital era muy avanzado y sus equipos eran muy obsoletos [...] (Moreno, 2008, pp. II8-119).

Finalmente, al entregarse las instalaciones del nuevo hospital, se hizo el traspaso a este de todos los bienes y equipamientos que pertenecían al San Juan de Dios. Legalmente, la substitución de una institución por otra, se hizo mediante el Acuerdo número 15 del 3 de diciembre de 1973, en el que se "autorizaba el reemplazo del antiguo hospital San Juan de Dios por las nuevas y modernas instalaciones del hospital RGV". Es así como se da la clausura definitiva del más antiguo y por muchos años único hospital público de la ciudad de Bucaramanga.

\section{VALORACIÓN Y CONCLUSIÓN}

Es posible afirmar que el antiguo hospital San Juan de Dios, el cual prestó sus servicios por más de 120 años, representa una parte importante del desarrollo histórico de Bucaramanga. Al ser por largos años la única institución pública prestadora de servicios de salud, cumplió un papel muy importante en procura de mejorar la calidad de vida y el bienestar de los bumangueses.

La calidad de institución de caridad y de beneficencia que caracterizó sus primeras décadas de vida al servicio de los más necesitados; la atención de los enfermos, en especial durante épocas en las que las epidemias azotaban a la población; así como el papel que jugó durante los acontecimientos históricos de la Batalla de Palonegro, son solo algunas de las razones para reconocer la relevancia del Hospital San Juan de Dios en la historia local.

Como institución, el San Juan de Dios representa también el esfuerzo que por varias generaciones los bumangueses tuvieron que hacer, participando activamente en la consecución de los recursos para su construcción y mantenimiento. Con el tiempo, el hospital fue ganándose un lugar dentro de las prioridades de los sucesivos gobiernos municipales, quienes por medio de contribuciones económicas intentaron mejorar las condiciones del servicio que por muchos años se caracterizaron por la ineficiencia, en razón de los escasos recursos económicos y humanos dispuestos para su funcionamiento.

Si bien la edificación en la cual alguna vez funcionó el San Juan de Dios se mantiene en pie y en aceptables condiciones estructurales, lo que no se puede garantizar en el tiempo. Por 
el momento no cuenta con algún tipo de declaratoria que lo proteja e impida una posible demolición. El vertiginoso crecimiento que se hace evidente en Bucaramanga en la actualidad y la necesidad de espacios para nuevas construcciones civiles o proyectos privados, pueden derivar en la pérdida de esta edificación que, como se intentó resaltar en este artículo, es parte importante de la historia de Bucaramanga.

\section{REFERENCIAS}

Acevedo, M. (1953). Centenario del Hospital San Juan de Dios de Bucaramanga. Revista Estudio, Año XXII, I8-2I, 23I-234.

Espinosa, C. (1999). Crecimiento urbanístico de Bucaramanga: 1850-1900. (Trabajo de grado para obtener el título de historiador). Bucaramanga, Universidad Industrial de Santander, Facultad de Ciencias Humanas, Escuela de Historia.

García, J.J. (1992). Crónicas de Bucaramanga. Bucaramanga: Imprenta del Departamento.

Harker, R. (1977). Y sucedió en Bucaramanga, vol. XXXI. Academia de Historia de Santander, Biblioteca Santander.

López, M.C., García, C., Medina, L.A. (2005). Manual para inventarios, bienes culturales inmuebles. Bogotá: Imprenta Nacional.

Ministerio de Cultura. (2009). Normas generales para la gestión, protección y salvaguardia del patrimonio cultural en Colombia. Bogotá: Autor.

Moreno, T.L. (2008). Historia de la salud pública en Bucaramanga 1920-1973. (Trabajo de grado para obtener el título de historiador). Bucaramanga: Universidad Industrial de Santander, Facultad de Ciencias Humanas, Escuela de Historia.

Spinel, J.F. (s.f.). El Centro de Bucaramanga. Aproximación interdisciplinaria para la renovación urbana. Informe final de pasantía. (Trabajo de grado para obtener el título de historiador). Bucaramanga: Universidad Industrial de Santander, Facultad de Ciencias Humanas, Escuela de Historia.

Valderrama, E. (1937). Monografía de Bucaramanga. Revista Estudio, año VI, 34-45.

\section{Fuentes}

Gaceta de Santander. (1870, noviembre 7). Año XII, pp. 509-5I0. Socorro.

Gaceta de Santander. (1888, septiembre 6). Año XXX, p. 493. Bucaramanga.

Revista Estudio. (1932, abril). Año II, Nº 8, p. 160. Bucaramanga.

Semanario El Posta. (1984, junio 23). Parque García Rovira. Serie II, No 27, p. 5.

Vanguardia Liberal. (1925, noviembre 27). Camas para el hospital, Año VII No. I897. Bucaramanga.

Vanguardia Liberal. (1953, marzo 10). Hace cien años nació el Hospital San Juan de Dios de Bucaramanga. 